\title{
The introduction of horizontal inhomogeneity of meteorological conditions in the EOSTAR propagation model
}

\author{
A.M.J. van Eijk* and G.J. Kunz \\ TNO Defence, Security and Safety, The Hague, The Netherlands,
}

\begin{abstract}
The effective field-of-view of an electro-optical sensor in a given meteorological scenario can be evaluated using a raytracer. The resulting ray trace diagram also provides information pertinent to the quality (distortion, mirages) of the image being viewed by the sensor. The EOSTAR (Electro Optical Signal Transmission And Ranging) model suite contains a ray tracer that has been upgraded to take into account horizontal inhomogeneities in the atmosphere, such as temperature gradients as observed in coastal areas where (e.g.) cold air flows out over warm waters. Initial results for horizontally inhomogeneous atmospheres are presented and compared to calculations for horizontally homogeneous atmospheres. It is shown that the horizontal inhomogeneity of temperature should be taken into account when assessing sensor performance.
\end{abstract}

Keywords: sensor performance, refraction, ray-tracer, horizontal inhomogeneity, EOSTAR

\section{INTRODUCTION}

Electro-optical (EO) systems are nowadays widely used for surveillance applications in the marine environment. They provide a useful complement to radar (RF) systems and since EO-systems are passive, their use may be preferred over active radar systems in certain situations. While the range of electro-optical systems is usually shorter than that of radar systems, their imaging capability proves useful for classification of contacts, which makes EO-sensors a useful complement to radar (RF) systems. Since EO-systems are passive, their use may even be preferred over active radar systems in certain situations. The maximum range of both RF and EO sensors is generally not determined by technological limitations, but by the prevailing meteorological conditions. The effects of the environment are wavelength (or frequency) dependent, which implies that varying meteorological conditions favor different sensors and/or frequency bands.

The atmosphere thus has an important effect on the propagation of electro-optical radiation. Molecules and aerosols in the atmosphere scatter and absorb radiation resulting in transmission losses, and hence a reduced range. In the marine environment, absorption by water vapor is a key process in range assessment for the long-wave IR band (8-12 $\mu \mathrm{m})$. Aerosols predominantly scatter radiation and are most pertinent to transmission in the short-wave IR band (3-5 $\mu \mathrm{m})$, near-IR or visible bands. Compared to molecules, the concentration (and the composition in coastal environment) of aerosols is a highly fluctuating quantity, which makes it difficult to assess their contribution to the transmission losses.

The second phenomenon affecting the propagation of electro-optical radiation is refraction. Refraction effects arise from variations in the vertical profiles of meteorological parameters such as humidity and temperature. A distinction is made between long-term, large-scale variations and short-term, small-scale variations. The latter variations are associated with turbulence in the atmosphere, and the resulting refraction effects are generally referred to as scintillation (intensity fluctuations) and blur (image distortion). The term 'refraction effects' is usually reserved for the effects of the long-term, large-scale variations of meteorological parameters, which often are much more drastic than scintillation and blur. Over a range of several kilometers, the large-scale refraction effects may cause significant deviations (ray-bending) of the propagation path as compared to situations where the refractive index of the air is invariant with height (straight line). In this manner, the optical horizon (maximum unobstructed range of sensor) may become smaller than the geometrical

\footnotetext{
* Contact information: lex.vaneijk@tno.nl, phone: +31 70374 0451, TNO Defence, Security and Safety, P.O. Box 96864, 2509 JG The Hague, The Netherlands; http://www.tno.nl
}

Atmospheric Optical Modeling, Measurement, and Simulation II, edited by Stephen M. Hammel, Anton Kohnle, Proc. of SPIE Vol. 6303, 63030G, (2006) · 0277-786X/06/\$15 · doi: 10.1117/12.680300 
horizon (expected maximum unobstructed range without refraction effects), which results in a delayed threat detection. The opposite is also possible resulting in earlier threat detection. In the radar community, such conditions are generally referred to as ducting. Since refraction effects are height-dependent, severe image deformation may occur for extended (not point source) targets. This obviously hampers the classification and identification process. The most dramatic manifestation of refraction effects is the presence of a secondary image (mirage) of a target on the detector while in reality there is only a single target. These mirages increase the false alarm rate of surveillance systems and hamper tracking of targets.

The effective detection, classification and identification ranges of electro-optical surveillance systems in a given meteorological scenario are important parameters in the assessment of both the vulnerability of a military platform as well as its strike capacity. To make this assessment, a tool is required that ideally has now- and forecasting capabilities of the electro-optical propagation environment. From the discussion above, it is evident that such a tool must integrate a variety of modules that govern the complete observation chain. This chain includes the background against which a target is viewed, the target, the atmosphere, the sensor and the signal processing. These comprehensive tools are referred to as system or end-to-end models.

Since 2001, TNO and Spawar Systems San Diego have been involved in the development of the EOSTAR ${ }^{1}$ (Electro Optical Signal Transmission And Ranging) model suite, which aims at providing an user-friendly end-to-end model. The suite consists of a computational toolbox, which can be embedded in a customized GUI (Graphical User Interface). In this manner, the suite may be optimized for specific applications, such as mission planning, platform vulnerability or the analysis of sensor acceptance trials. Typical output consists of the synthetic sensor image ("how does the target appear?") or a coverage diagram ("where can I detect / classify the target?"). The EOSTAR model suite has been presented in various contributions. ${ }^{2-4}$

In this paper, we address the aspect of horizontal inhomogeneity of the atmosphere and the impact thereof on the EOSTAR predictions. Like many propagation codes, the initial versions of EOSTAR assume that the atmosphere is horizontally homogeneous. For typical EO-ranges (say, up to 25 miles) this assumption may be valid in open ocean conditions, but it is likely to fail in the coastal zone. The coastal zone is characterized by strong horizontal inhomogeneity as a result of the land-sea interface. In off-shore flow conditions, warm air may be advected out over a relatively cold sea (or cold air over a warm sea), resulting in strong temperature gradients over a relatively short distance. Thus, there is a need to assess the effect of these gradients on the propagation conditions and in particular on the refractive phenomena. The next section discusses the modules of EOSTAR which govern the calculations of the ray trajectories. Section 3 discusses the recent upgrade of EOSTAR, ${ }^{*}$ capable of taking horizontal inhomogeneity into account. Initial results of the upgraded EOSTAR are presented in section 4.

\section{CALCULATION OF RAY TRAJECTORIES}

EOSTAR builds a marine atmospheric surface layer on the basis of simple meteorological input parameters using the well-known Monin-Obukhov similarity theory. ${ }^{5}$ The relevant module in EOSTAR is the TARMOS (Turbulence And Refraction Modeling Over the Sea) model. ${ }^{6}$ TARMOS can be used in two modes: with an assumed relation for $z_{0 m}$, the roughness length for momentum, ${ }^{7}$ or with an assumed relation for $C_{D N}$, the drag coefficient under neutral conditions. ${ }^{8}$ The output of TARMOS consists of the (logarithmic) vertical profiles of wind speed, temperature and water vapor, as well as characteristic turbulence (micrometeorological) quantities. The vertical profiles are subsequently used to calculate the vertical profile of refractive index.

The long-term, large-scale refraction effects are calculated by a ray tracer based on Snell's law for spherically layered structures, which calculates the actual trajectory of electro-optical radiation propagating through the atmosphere. The basic parameter of the ray tracer is the index of refraction $n$ of the atmosphere, which is wavelength dependent. More often the refractivity $N=(n-1) \cdot 10^{6}$ is used, since the variations of $n$ in the atmosphere are quite small. Many authors have reported (approximate) relations to infer the refractivity as function of atmospheric conditions. For visible and infrared radiation, Edlé ${ }^{9,10}$ has made pioneering efforts, resulting in the following model: ${ }^{11}$

\footnotetext{
* For this study, the ARTEAM calculation kernel was used.
} 


$$
N(v)=A(v) \cdot \frac{P}{P_{0}} \cdot \frac{\left(T_{0}+15\right)}{T}-C(v) \cdot \frac{P_{w}}{P_{0}}
$$

where $\mathrm{P}_{0}=1013.35 \mathrm{hPa}, \mathrm{T}_{0}=273.15 \mathrm{~K}$ and

$$
A(v)=\left[83.42+\frac{185.08}{1-\left(v / 1.14 \cdot 10^{5}\right)^{2}}+\frac{4.11}{1-\left(v / 6.24 \cdot 10^{4}\right)^{2}}\right] \quad ; \quad C(v)=\left[43.49-\left(\frac{v}{1.7 \cdot 10^{4}}\right)^{2}\right]
$$

The variables in eq.(1) are: $v$ the frequency in $\left[\mathrm{cm}^{-1}\right], T$ the air temperature in $[\mathrm{K}]$ and $P_{w}$ the water vapor pressure in [hPa]. The latter quantity can be obtained from the temperature and the (absolute or relative) humidity. A sensitivity analysis of eq.(1) shows that $N(v)$ is mainly dependent on air temperature and pressure, and that water vapor plays a relatively small role at visible and infrared wavelengths. Eq.(1) allows calculating the vertical profile of refractive index, using temperature and humidity as provided by TARMOS and pressure as obtained from the hydrostatic equation.

Ray trajectories in the Earth' atmosphere can basically be constructed from successive application of Snell's law on the interface of two layers, each with their own index of refraction. However, this approach requires extra steps due to the curvature of the Earth. Alternatively, the spherically layered structure can be transformed to a horizontally layered one in which the refractive index transforms to the so-called modified refractivity, which is a function of the refractive index and the radius of the Earth curvature. A third and direct approach, which we use in our ray tracer, is the application of Snell's law for spherically layered structures ${ }^{12}$, which is given by:

$$
n_{1} R_{1} \sin \left(\varphi_{1}\right)=n_{2} R_{2} \sin \left(\varphi_{2}\right)
$$

With equation (3), the variation in ray direction can be expressed in terms of the first derivative of the refractive index with height and the ray can be constructed relative to the center of the Earth.

The trajectory of a ray is ended when it hits the surface. In the marine environment, the surface consists of waves and their amplitude must be taken into account when evaluating the ray trajectory. Recently, a version of EOSTAR was presented that takes into account the statistics of the wave amplitudes. ${ }^{13}$ However, for this initial study on horizontal inhomogeneity the wave amplitude was set to a constant height and rays were ended when descending below this height.

\section{HORIZONTAL INHOMOGENEITY IN EOSTAR}

To introduce horizontal inhomogeneity in EOSTAR, a spatial (X-Y), rectangular grid was defined. The grid spacing in East-West and North-South directions can be varied, and meteorological conditions can be defined at each grid point. Typical grid point spacings are of the order of $5-10 \mathrm{~km}$. This lay-out prepares EOSTAR for meteorological input as provided by mesoscale meteorological models, such as COAMPS (Coupled Ocean/Atmosphere Mesoscale Prediction System ${ }^{14}$ or WRF (Weather Research and Forecasting model). ${ }^{15}$ Coupling EOSTAR to such models will greatly extend the applicability of the model and allows also to increase the vertical domain above the marine surface layer.

The sensor is then positioned in the master grid defined above. Along the sensor radial (i.e., viewing direction), a secondary grid is defined. Again, the grid spacing can be varied (typical spacing is 1-5 km). At each grid point along the radial, the meteorological conditions are calculated from an interpolation of the master grid. Subsequently, TARMOS is run for each secondary grid point and a refractive index profile is generated for each secondary grid point. Finally, rays are traced from the sensor. At each step (typically of the order of 50 meters) along the trajectory, the refractive index is calculated by an interpolation of the two nearest grid points on the secondary grid.

It could be argued that it is more proper to only interpolate the individual values of temperature, humidity and pressure and to defer the calculation of the refractive index to the very last moment, i.e., at the exact position of the ray trajectory. This allows to more accurately taking into account the individual gradients in the basic meteorological parameters. 

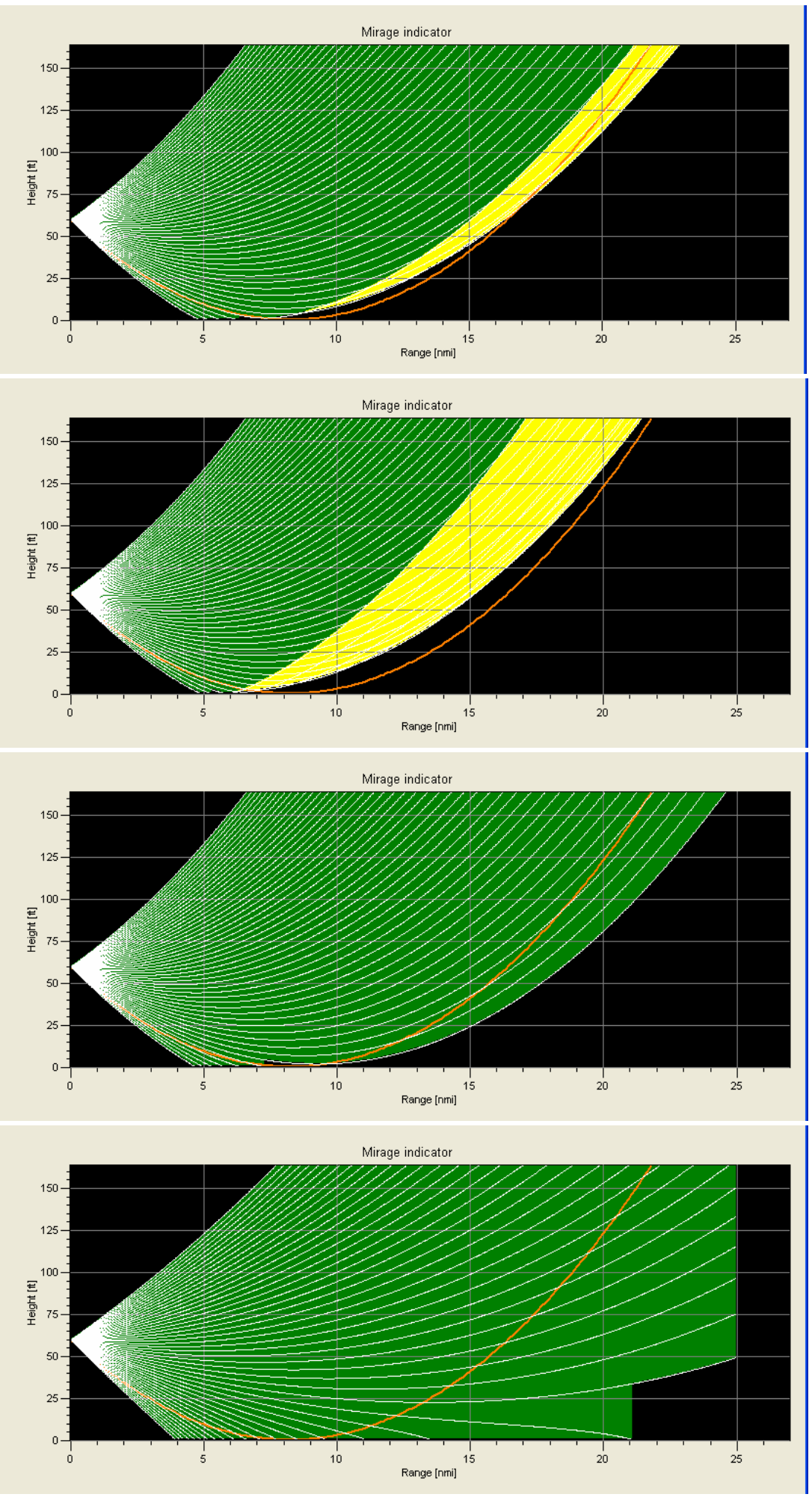

(Panel 1)

(Panel 2)

(Panel 3)

(Panel 4)

Figure 1: Horizontal inhomogeneity in temperature. Panel (1): gradient from 7 to $12^{\circ} \mathrm{C}$; Panel (2): constant temperature of $7^{\circ} \mathrm{C}$; Panel (3): constant temperature of $9.5^{\circ} \mathrm{C}$; Panel (2): constant temperature of $12^{\circ} \mathrm{C}$; see text for further explanation. 
However, the EOSTAR code structure does not easily allow for such a calculation and this venue has not further been explored. Furthermore, the mapping of the meteorological parameters on the secondary grid accounts at least partially for the individual spatial gradients.

\section{INITIAL RESULTS}

For the first test with horizontal inhomogeneity, the relevant meteorological conditions were sea temperature of $9^{\circ} \mathrm{C}$, relative humidity of $80 \%$ at $12 \mathrm{~m}$ height and air pressure of $1000 \mathrm{hPa}$ at $12 \mathrm{~m}$ height. The air temperature was also specified at $12 \mathrm{~m}$ height. Three runs have been made with horizontally homogeneous atmospheres, i.e., for air temperatures of $7^{\circ} \mathrm{C}$ (panel 2 in figure 1), of $9.5^{\circ} \mathrm{C}$ (panel 3 in figure 1) and of $12^{\circ} \mathrm{C}$ (panel 4 in figure 1). Then, a run was made with a horizontal temperature gradient ranging from $7^{\circ} \mathrm{C}$ at the sensor's position to $12^{\circ} \mathrm{C}$ at 25 nautical miles from the sensor (panel 1 in figure 1).

Figure 1 shows ray tracer and coverage diagrams for the sensor under consideration. The sensor is a generic thermal imager, operating in the $4-5 \mu \mathrm{m}$ wavelength band, with 50 vertical pixels and a vertical field-of-view (FOV) of 4.56 $\mathrm{mrad}$. The sensor is positioned at a height of 60 feet at range 0 (left side of figure 1). Figure 1 presents the ray trajectories for each sensor pixel. The ray tracer automatically ends a ray trajectory after 25 nautical miles, hence the abrupt ending at the righthand side of panel 4 .

The colored area between the rays constitutes the FOV of the sensor. In other words, the sensor may only see a target when the target is located within the FOV. Whether the target is actually detected, depends on additional factors such as target contrast and signal processing. Furthermore, mirages may occur where the individual ray trajectories cross: in that case there are two (or more) possible pathways from target to observer, resulting in two different angles under which the same (part of the) target can be viewed. The coverage diagram in figure 1 indicates the presence of mirage zones by a yellow (lightgray) color (see panels 1 and 2), which may warn the operator for possible difficulties in recognition and classification, as well as an increased false alarm rate.

Finally, an orange line in figure 1 indicates the location of the geometric horizon, i.e., the location of the horizon in a non-refracting atmosphere. The horizon line is curved, because figure 1 uses the "flat earth representation" in which the earth's curvature has been eliminated from the vertical axis. Panels 3 and 4 show that, when the air is warmer than the sea ${ }^{\dagger}$ 'the sensor is able to 'see' beyond the geometric horizon, which could potentially result in earlier detection of threats. On the contrary, when the air is colder than the sea (panel 2), the sensor's potential range is shorter than the geometric horizon.

Panel 1 in figure 1 shows the results for a temperature gradient of $5^{\circ} \mathrm{C}$ over $25 \mathrm{nmi}$. The figure was obtained with a master grid size of $5 \mathrm{nmi}(9.2 \mathrm{~km})$ and a secondary (along sensor radial) grid spacing of $5 \mathrm{~km}$ (see previous section). A number of larger and smaller grid sizes were tested, but the results shown in panel 1 of figure 1 did not change as function of grid choice.

A comparison of panel 1 (temperature gradient of $5^{\circ} \mathrm{C}$ over $25 \mathrm{nmi}$ ) and the other panels in figure 1 reveals that the results obtain for the horizontally inhomogeneous atmosphere differ considerably from the results obtained with homogeneous atmospheres of the extreme $\left(7\right.$ or $\left.12^{\circ} \mathrm{C}\right)$ or average $\left(9.5^{\circ} \mathrm{C}\right)$ temperatures. If one focuses on the mirage zone, panel 2 (constant temperature of $7^{\circ} \mathrm{C}$ ) is the only one that also predicts a mirage zone, but its width is much larger. Furthermore, panel 1 shows that for lower altitudes (below 50 feet), the sensor's FOV does not quite reach the geometric horizon, whereas for higher altitudes the sensor can 'see' somewhat beyond the horizon. In contrast with this, the sensor's FOV in panel 2 does not reach the geometric horizon at any altitude. Thus, it is not very appropriate to use the extreme or average temperatures to approach the diagrams found for a temperature gradient.

By trial-and-error, the best match between panel 1 and a homogeneous atmosphere was found for a (constant) temperature of $8.55^{\circ} \mathrm{C}$. Not surprisingly, this is approximately the temperature that the inhomogeneous atmosphere has at

\footnotetext{
$\dagger$ This is a somewhat simplified statement, since the relevant criterion is atmospheric stability that depends on temperature, water vapor and wind speed.
} 


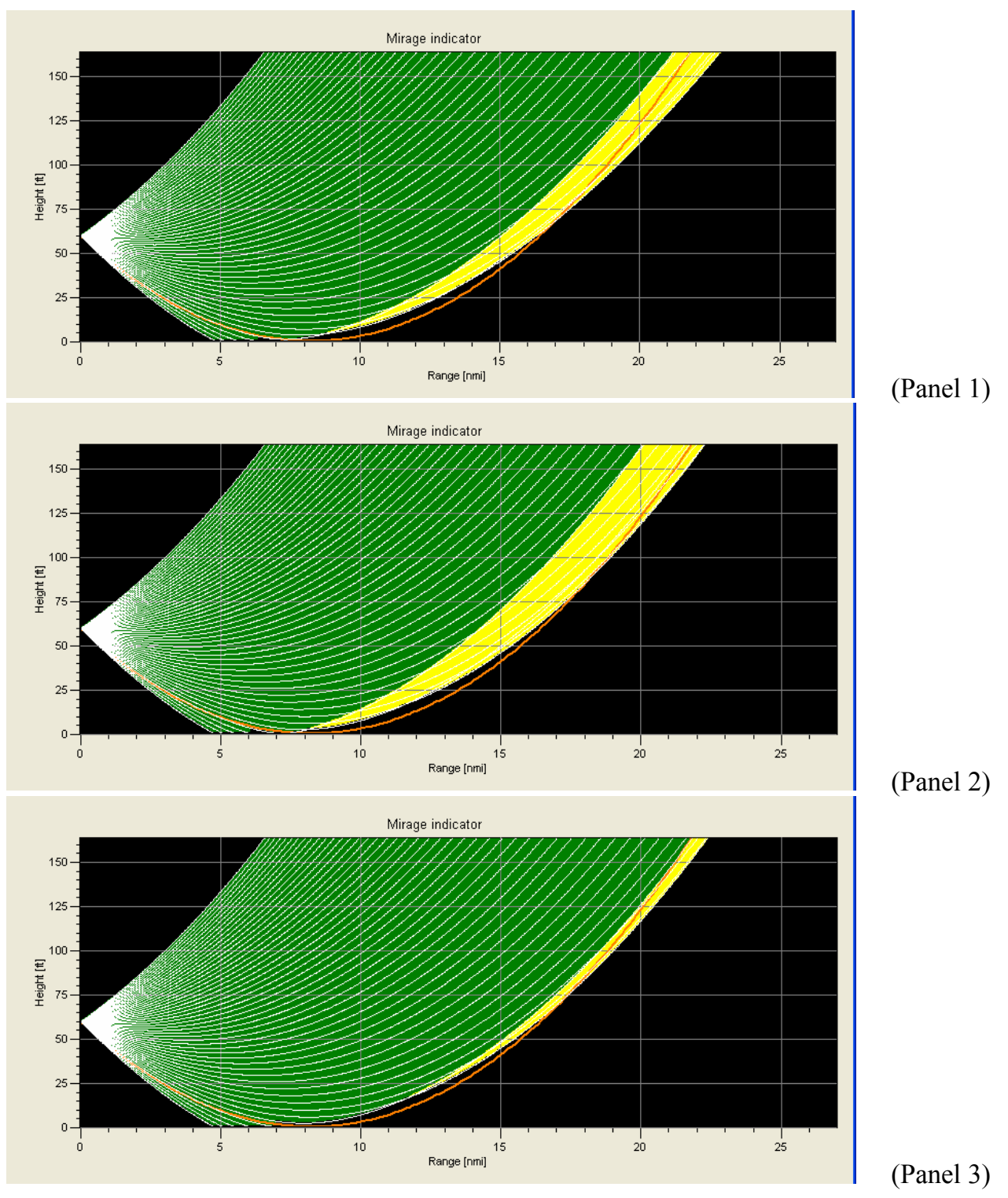

Figure 2: Horizontal inhomogeneity in temperature. Panel (1): gradient from 7 to $12^{\circ} \mathrm{C}$ (identical to figure 1, panel 1); Panel (2): constant temperature of $8.55^{\circ} \mathrm{C}$; Panel (3): constant temperature of $8.65^{\circ} \mathrm{C}$.

7.5 nautical miles from the original and where the ray bending is most significant. This area of significant ray bending determines to a large extent the characteristics of the mirage zone. Nevertheless, figure 2 shows that mirage zones for this homogeneous $\left(8.55^{\circ} \mathrm{C}\right)$ and inhomogeneous $\left(7-12^{\circ} \mathrm{C}\right.$ gradient $)$ differ at higher altitudes, where the rays have passed relatively long distances through atmospheres with different temperatures (constant $8.55^{\circ} \mathrm{C}$ versus $8.5^{\circ} \mathrm{C} @ 7.5 \mathrm{nmi}$ increasing to about $11.5^{\circ} \mathrm{C} @ 22.5 \mathrm{nmi}$ ). However, panels 1 and 2 demonstrate that if one does not have an algorithm for inhomogeneous temperatures, a run for a homogeneous atmosphere with the temperature at approximately the range of strongest ray-bending may provide a good indication. It is not evident to judge the exact temperature that should be used in the homogeneous atmosphere. Panels 3 shows the mirage zone calculated for a homogeneous atmosphere with a temperature of $8.65^{\circ} \mathrm{C}$ : already, the match with the inhomogeneous atmosphere (panel 1) is significantly less. 


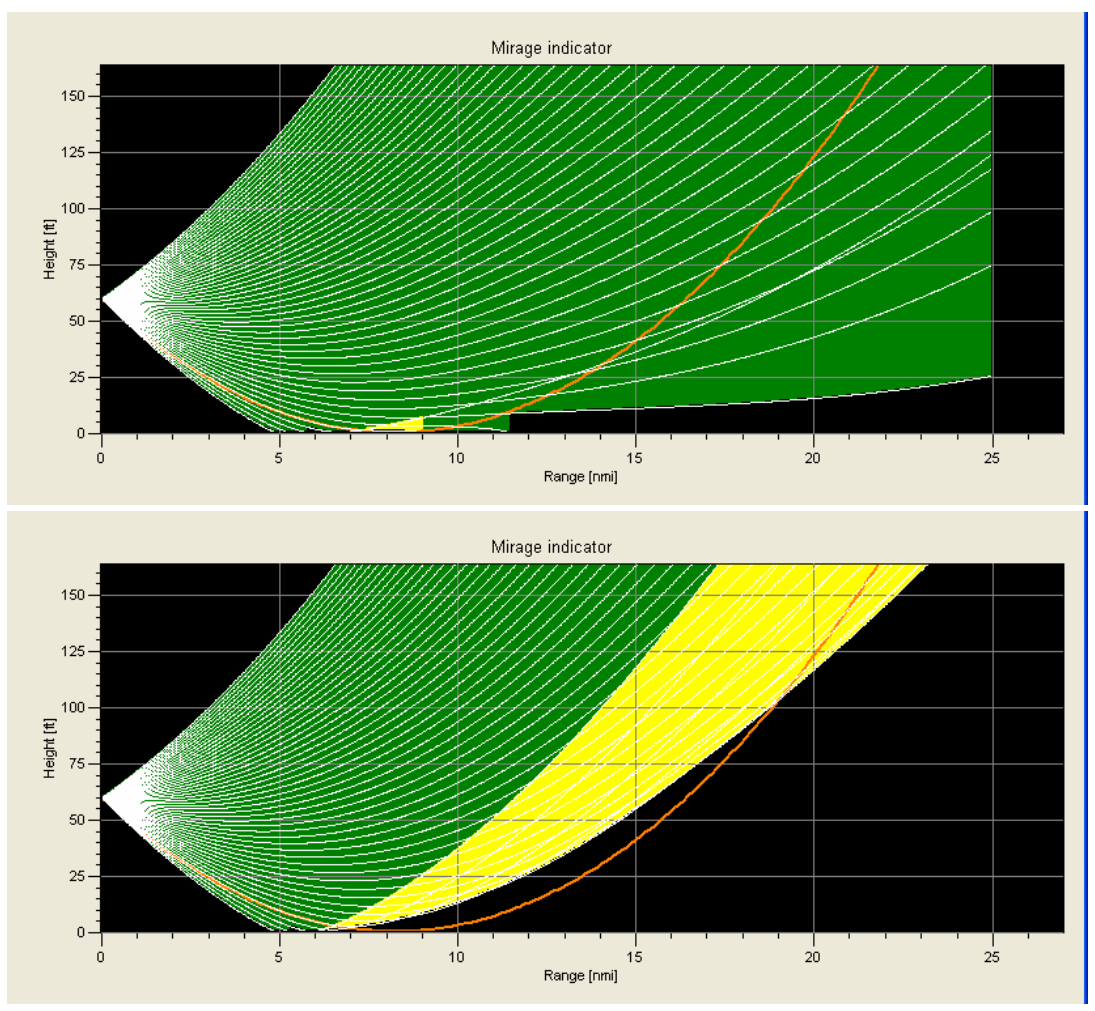

(Panel 1)

(Panel 2)

Figure 3: Horizontal inhomogeneity in temperature. Panel (1): gradient from 7 to $12^{\circ} \mathrm{C}$ between 5 and 10 miles range; Panel (2): gradient from 7 to $12^{\circ} \mathrm{C}$ between 10 and 15 miles range

Finally, a few tests were made for an extreme case in which the temperature jump was confined to a relatively small part of the horizontal domain. Figure 3 shows the ray trajectories for an atmosphere with a temperature jump from $7^{\circ} \mathrm{C}$ to $12^{\circ} \mathrm{C}$ between 5 and 10 nautical miles (top panel) and between 10 and 15 nautical miles (bottom panel). These ray trajectory diagrams cannot easily be approximated with the code for homogeneous atmospheres and a single temperature.

Similar tests as described above have been completed with gradients of relative humidity ( 70 to $90 \%$ over $25 \mathrm{nmi}$ ) and pressure (1000 to $1010 \mathrm{hPa}$ over $25 \mathrm{nmi})$. In the case of pressure, no significant differences between inhomogeneous or homogeneous atmospheres were observed. The refractive index is only weakly dependent on pressure, and the pressure variation was too small to affect the ray trajectories. For the gradient of relative humidity, an effect was observed, albeit less striking than for the gradient of temperature. The same conclusions as given above for temperature gradients pertain to the relative humidity case.

The development of EOSTAR for inhomogeneous atmosphere has not yet been completed. The next step involves introducing the horizontal inhomogeneity in the calculation of molecular and aerosol transmission, path and background radiance. This will allow us to more correctly calculate the contrast of a target as seen by the sensor.

\section{ACKNOWLEDGEMENTS}

Parts of this work have been sponsored by grants from The Royal Netherlands Navy (program V509, Environmental effects on Sensor and Weapons systems, Mr. Wim Pelt) and the US Office of Naval Research by contract N68171-05-P0147 (Dr Ron Ferek). 


\section{REFERENCES}

1. See: http://www.tno.nl/eostar.

2. G.J. Kunz, M.M. Moerman and A.M.J. van Eijk. ARTEAM : Advanced Ray Tracing with Earth Atmospheric Models. SPIE Proceedings 4718, 397-404, 2002.

3. G.J. Kunz, M.M. Moerman, A.M.J. van Eijk, S.M. Hammel and D. Tsintikidis (2003). EOSTAR : An electro-optical sensor performance model for predicting atmospheric refraction, turbulence and transmission in the marine surface layer. SPIE Proceedings 5237, 81-92, 2003.

4. G.J. Kunz, A.M.J. van Eijk, S.M. Hammel and D. Tsintikidis. EOSTAR: A System Performance Tool for the Marine Near-Surface Environment. Battlespace Atmospheric and Cloud Impacts on Military Operations (BACIMO) conference, Monterey CA, USA, 12-14 October 2005.

5. A.S. Monin and A.M. Obukhov, "Basic laws of turbulent mixing in the atmosphere near the ground", Tr. Inst. Acad. Nauk. Sci., SSR. Inst. Geophiz., 24, 1963-1987, 1954.

6. G.J. Kunz, "A bulk model to predict optical turbulence in the marine surface layer", TNO Physics and Electronics Laboratory, The Hague, The Netherlands, report FEL-96-A053, 1996.

7. Smith, S.D., "Coefficients for sea surface wind stress, heat flux and wind profiles as a function of wind speed and temperature", J. Geophys. Res. 93, 15,467-15,472, 1988.

8. K.L. Davidson, G.E. Schacher, C.W. Fairall, and A.K. Goroch, "Verification of the bulk method for calculating overwater optical turbulence", Appl. Opt. 20, 2919-2924, 1981.

9. B. Edlén, "The dispersion of standard air", J. Opt. Soc. Am. 43, 339-343, 1953.

10. B. Edlén, "The refractive index of air", Meteorol. 2, 71-80, 1966.

11. R.W. Fenn, S.A. Clough, W.O. Gallery, R.E. Good, F.X. Kneizyz, J.D. Mill, L.S. Rothman, E.P. Shettle, F.E. Volz, "Optical and infrared properties of the atmosphere", in Handbook of geophysics and space environment edited by A. S. Jursa, Air Force Geophysics Laboratory, Air Force Systems Command, USA, 18.1-18.80, 1985.

12. W.G. Rees, C.M. Roach, and C.H.F. Glover, "Inversion of atmospheric refraction data", J. Opt. Soc. Am. $\underline{8}$, 330338, 1991.

13. M. J. Francius, G. J. Kunz, and A. M. J. van Eijk. Surface wave effects on long range IR imaging in the marine surface layer. SPIE Proceedings 5891, 2005.

14. http://www.nrlmry.navy.mil/coamps-web/web/home.

15. http://www.wrf-model.org/index.php. 